\title{
Advanced Control Considerations for Turbofan Engine Design
}

\author{
Joseph W. Connolly* \\ NASA Glenn Research Center, \\ Cleveland, $\mathrm{OH}$ 44135, USA
}

\author{
Jeffrey T. Csank ${ }^{\dagger}$ \\ NASA Glenn Research Center, \\ Cleveland, $\mathrm{OH}$ 44135, USA
}

Amy Chicatelli ${ }^{\ddagger}$

Vantage Partners LLC, Brook Park, OH 44142, USA

\begin{abstract}
This paper covers the application of a model-based engine control (MBEC) methodology featuring a self tuning on-board model for an aircraft turbofan engine simulation. The nonlinear engine model is capable of modeling realistic engine performance, allowing for a verification of the advanced control methodology over a wide range of operating points and life cycle conditions. The on-board model is a piece-wise linear model derived from the nonlinear engine model and updated using an optimal tuner Kalman Filter estimation routine, which enables the on-board model to self-tune to account for engine performance variations. MBEC is used here to show how advanced control architectures can improve efficiency during the design phase of a turbofan engine by reducing conservative operability margins. The operability margins that can be reduced, such as stall margin, can expand the engine design space and offer potential for efficiency improvements. Application of MBEC architecture to a nonlinear engine simulation is shown to reduce the thrust specific fuel consumption by approximately $1 \%$ over the baseline design, while maintaining safe operation of the engine across the flight envelope.
\end{abstract}

Nomenclature

System matrix

System matrix

System matrix

System matrix

System matrix

Kalman Filter gain

System matrix

System matrix

Covariance matrix of

estimated parameters

Process noise covariance matrix

Transformation matrix relating

health and tuning parameters

Error

Health parameter vector

Kalman Filter tuning

parameter vector

$\begin{array}{ll}r & \text { Control setpoint } \\ u & \text { Actuator command vector } \\ v & \text { Measurement noise vector } \\ w & \text { Process noise vector } \\ x & \text { State vector } \\ y & \text { Measured outputs vector } \\ z & \text { Estimated outputs vector }\end{array}$

Subscript

c Control parameter

$x h \quad$ Augmented state vector $(x$ and $h)$

$x q \quad$ Reduced order State vector $(x$ and $q)$

$\begin{array}{ll}\hat{S}^{\text {Superscripts }} & \\ \dagger & \text { Estimated value } \\ & \text { Pseudo-inverse }\end{array}$

*Aerospace Engineer, Intelligent Controls and Autonomy Branch, 21000 Brookpark Rd., 77-1, AIAA Senior Member

$\dagger$ Aerospace Engineer, Intelligent Controls and Autonomy Branch, 21000 Brookpark Rd., 77-1, AIAA Senior Member

¥Aerospace Engineer, 3000 Aerospace Parkway VPL-3, AIAA Senior Member 


\section{Introduction}

$\mathrm{T}^{\mathrm{N}}$ current engine control architectures, the typical design approach is to regulate a measurable variable

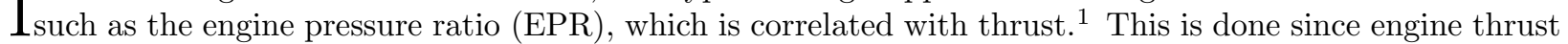
is not a measurable parameter from typical on-board engine sensors. Additionally, limits on allowable fuel flow are imposed based on a conservative approach to maintain an adequate stall margin (SM) constraint for safe operation of the engine throughout its life. ${ }^{2}$ Stall margin is defined as a measure of the distance from the operating line to the stall line on a compressor performance map, shown in Fig. 1. While engine stall is a serious engine phenomena that needs to be avoided, ${ }^{3}$ the existing control approaches result in overly conservative engine operation and can lead to lower efficiency than otherwise would be obtained.

Model-based engine control (MBEC) is being developed as an advanced control system methodology to improve turbofan engine efficiency. The development of MBEC, as applied to commercial turbofan engines, has evolved over the past decades towards a more "Intelligent" engine that can tailor itself to specific user requirements. ${ }^{4-7}$ The realization of a more customizable engine is obtained through the use of an on-board model designed to provide real-time estimates of desired unmeasured parameters such as thrust, combustor exit temperature, and SM. MBEC can allow operation with less conservative operability margins, since operability margins are typically designed for an end of life (EoL) engine, whereas an on-board model can provide a more accurate margin for the actual condition of the engine. The longer term pay-off of this research is to have personalized control for each specific engine. Personalized control to the actual condition of the engine will enable engine design to maintain more efficient operation throughout its lifetime, and also to make adjustments to meet specific mission requirements. ${ }^{8,9}$

A generic compressor performance map illustrating the SM is shown in Fig. 1. The SM constraint consists of two main components. First, there is an uncertainty margin that allows for steady state operation of the engine throughout its operating envelope and life cycle. Second, there is a transient margin that allows the engine to maneuver from one point on the operating line to another steady state operating point. Modifications to the operating line (green) can be obtained by trading components of the operability margin, such as uncertainty and transient manuverability (blue) to move the operating line closer to the stall line (red). This allows the operating line to be adjusted to higher pressure ratios and potentially more efficient portions of the performance map with the goal of obtaining efficiency gains. A stall margin limiter can be developed to ensure that a lower SM threshold can be used for developing a new operating line while maintaining safe operation.

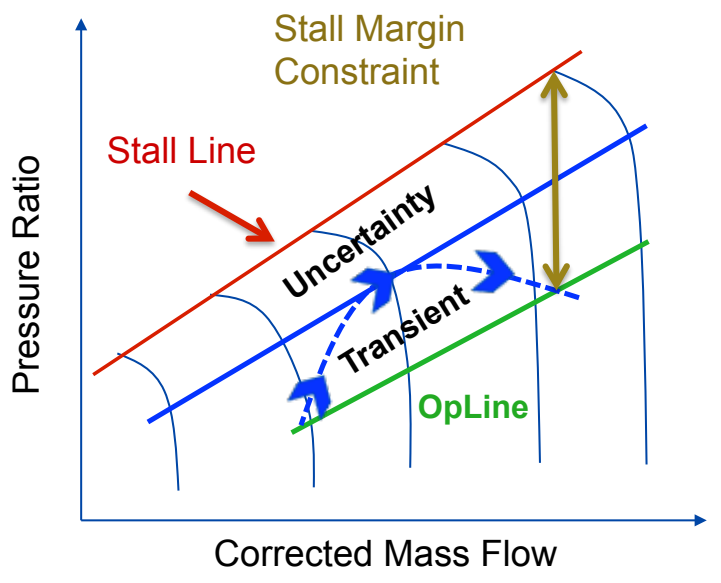

Figure 1. Generic compressor performance map illustrating stall margin constraint.
The focus here is on reducing the turbofan engine thrust specific fuel consumption (TSFC), which has typically been accomplished through the development of technologies focused on the optimization of the steady state operation, where advanced controls technologies have not had a significant role. Controls development for a turbofan engine is usually conducted once the steady steady state engine cycle is nearly finalized. In this study, MBEC is used to improve the protection logic for the high pressure compressor (HPC) SM as it plays an important role in designing the engine operating line. ${ }^{10}$ Initially, studies were conducted to move the operating line through the current control actuators; however, this did not provide sufficient capability to change the operating line. This prompted the investigation of redesigning the turbine component of the engine. By making modifications to the operating line through an engine redesign that includes controls, the goal of obtaining efficiency gains can be realized by operating at higher pressure ratios closer to the stall line. This paper builds upon previous work that developed the MBEC architecture. ${ }^{11}$

The paper is organized as follows. First, the current baseline engine control architecture is introduced followed by the MBEC control architecture used in this approach. Next, an application of the MBEC architecture to modify engine design is provided. Results are presented to compare an engine model with the baseline controller to that of the redesigned engine model with the MBEC architecture. Finally, concluding remarks are presented. 


\section{Baseline Control Architecture}

A baseline control architecture for a turbofan engine equipped with a full authority digital engine control is shown in Fig. 2. The control system in a modern commercial aircraft engine is designed to operate the engine in a conservative and safe manner throughout its operating envelope during its on-wing life. Turbofan engine controllers are designed to regulate a measurable variable such as EPR, which is correlated with thrust. This is done since there is no on-board sensor for thrust. The main interface between the pilot and the control system is the power lever angle (PLA) or throttle, which is used by the controller logic to set a required EPR demand. The measured EPR is subtracted from the command set point to create an error signal that goes to the controller to determine a fuel flow rate $\left(W_{f}\right)$. Protection logic limits the commanded fuel flow rate to provide safe operation of the engine. Finally, the fuel flow signal is sent to the fuel metering valve. For safety and operability, protection logic is used to regulate excessive transients and other operating limits that could lead to stall or other engine failures, as shown in Fig. 2. This is done by monitoring sensed parameters for the engine shafts' acceleration, combustor pressure, maximum fan shaft $\left(N_{f}\right)$ and core shaft $\left(N_{c}\right)$ speeds, and the ratio unit (RU), which is $W_{f}$ over compressor discharge static pressure. The acceleration of the engine shafts' serves as a proxy for SM since there is no on-board sensor for SM. The final $W_{f}$ signal that is provided to the fuel metering valve is the outcome of a selection process using a $\min / \max$ approach.

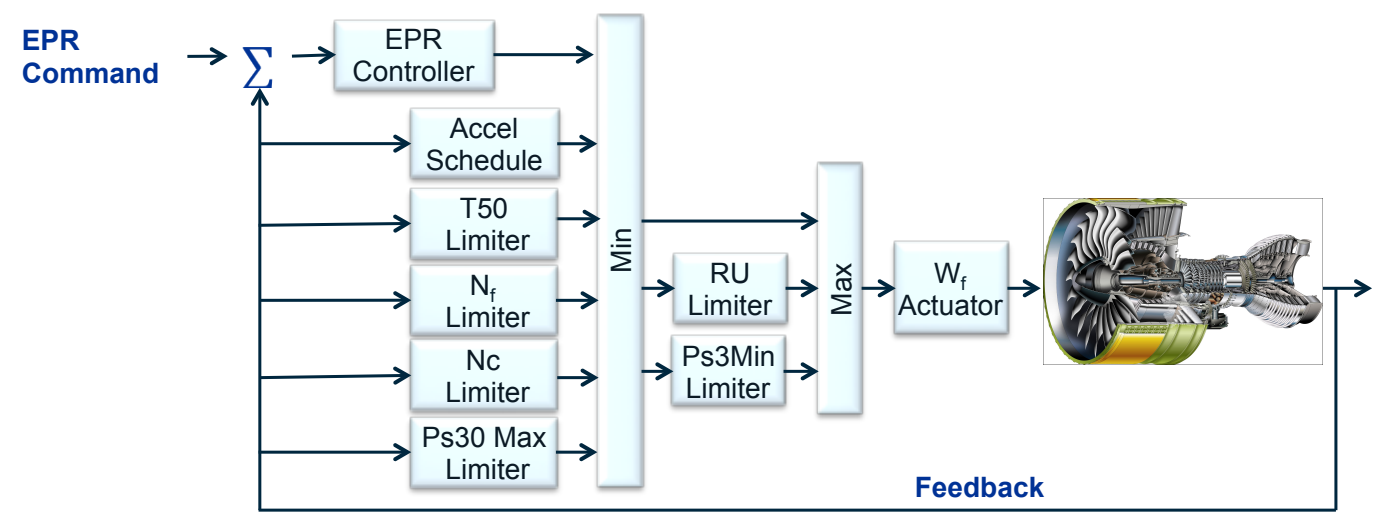

Figure 2. Baseline full authority digital controller diagram with a min/max protection logic.

\section{MBEC Control Architecture}

The use of on-board estimation of engine parameters to improve control and reduce conservative operation has a long history. ${ }^{4}$ The primary benefit explored here is in the reduction in engine SM conservatism. ${ }^{12,13}$ In the aircraft engine research community, MBEC is recognized as a means to provide the necessary improved efficiency to reduce engine-specific fuel consumption. ${ }^{5,7,14,15}$ The advancement proposed in this paper compared to previous work is the improved estimation across the engine life cycle, and the direct connection of controls into the engine design process.

A general schematic of the MBEC architecture applied in this study is shown in Fig. 3. A MBEC architecture is comprised of three main components; first an engine or "truth" model, second an on-board engine model, and last a controller with limit protection logic. For the simulation study presented in this paper, the Commercial Modular Aero-Propulsion System Simulation 40,000 (CMAPSS40k) ${ }^{16}$ will serve as the "truth" model engine. The MBEC application employs an optimal tuner Kalman Filter (OTKF) applied to a piece-wise linear model, which is derived from CMAPSS40k. This on-board model based control provides estimates of thrust and stall margin, and a thrust controller with stall margin protection.

In MBEC, sensed parameters of the baseline controller are replaced with estimations of key engine parameters of interest that do not have an on-board sensor. Thus, the EPR regulation is replaced by regulation of net thrust (Fnet). The safety limiters for acceleration protection and engine gas turbine temperature (T50) shown in Fig. 2 are replaced by a direct HPC SM limiter and combustor exit temperature (T40) limiter as shown in Fig. 3. The primary changes from the baseline controller are highlighted in yellow. Within the on-board self-tuning engine model, an OTKF estimation routine is used to self-tune the on-board 
model to the current engine condition. The following subsection will go into further detail of each of the three main components that comprise the MBEC architecture.

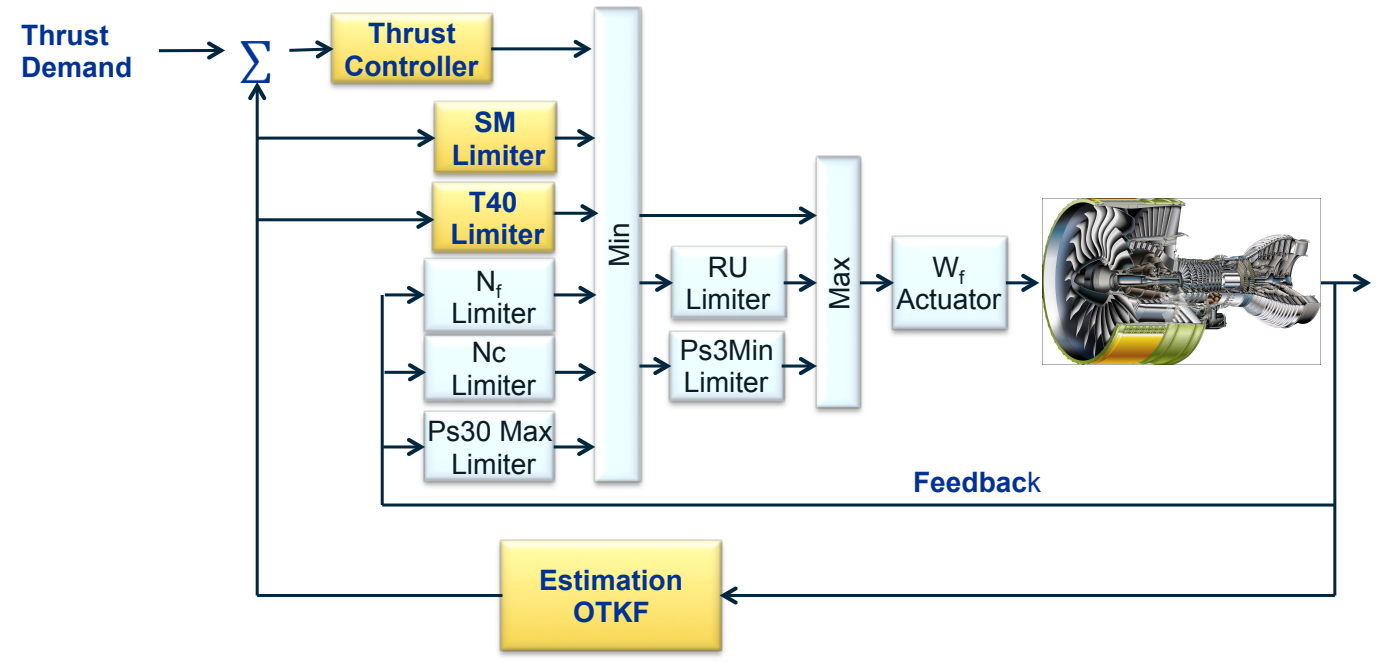

Figure 3. MBEC architecture with $\min / \max$ protection logic.

\section{A. Nonlinear Engine Simulation}

The CMAPSS40k simulation is a 40,000 lbf class turbofan engine simulation that is specifically designed for the development and testing of control algorithms. A general engine schematic with station numbers is shown in Fig. 4. The simulation provides realistic transient engine responses and is capable of executing faster than real time. Baseline controllers are provided in CMAPSS40k, along with protection logic shown in Fig. 2. For the CMAPSS40k simulation, the feedback controller and limiters are designed as gain scheduled proportionalintegral (PI) control with integrator windup protection. ${ }^{2}$ CMAPSS40k, in addition to controlling the fuel flow, also schedules the variable stator vane (VSV) and variable bleed valve (VBV).

The model contains a typical suite of sensors for turbofan engine control architectures, which includes:

1. $\mathrm{N}_{\mathrm{c}}$, core speed

2. $\mathrm{N}_{\mathrm{f}}$, fan speed

3. P25, inlet pressure

4. T25, inlet temperature

5. Ps30, high pressure compressor discharge static pressure

6. T30, high pressure compressor discharge temperature

7. T50, low pressure turbine exit temperature

8. P50, low pressure turbine exit pressure

The CMAPSS40k simulation contains a fleet average profile of engine deterioration versus number of flight cycles. By changing health parameters based on the percent deterioration of the engine, various stages of the engine life cycle can be simulated ranging from a $0 \%$ (new engine) to $100 \%$ (EoL engine). The health parameters account for deterioration percentage through the modification of the efficiency and flow capacity in each of the following engine components: fan, low pressure compressor (LPC), high pressure compressor (HPC), high pressure turbine (HPT), and low pressure turbine (LPT). For the transient simulations to follow it should be noted that the PLA has a range of 40 degrees (flight idle) to 80 degrees (maximum thrust). 


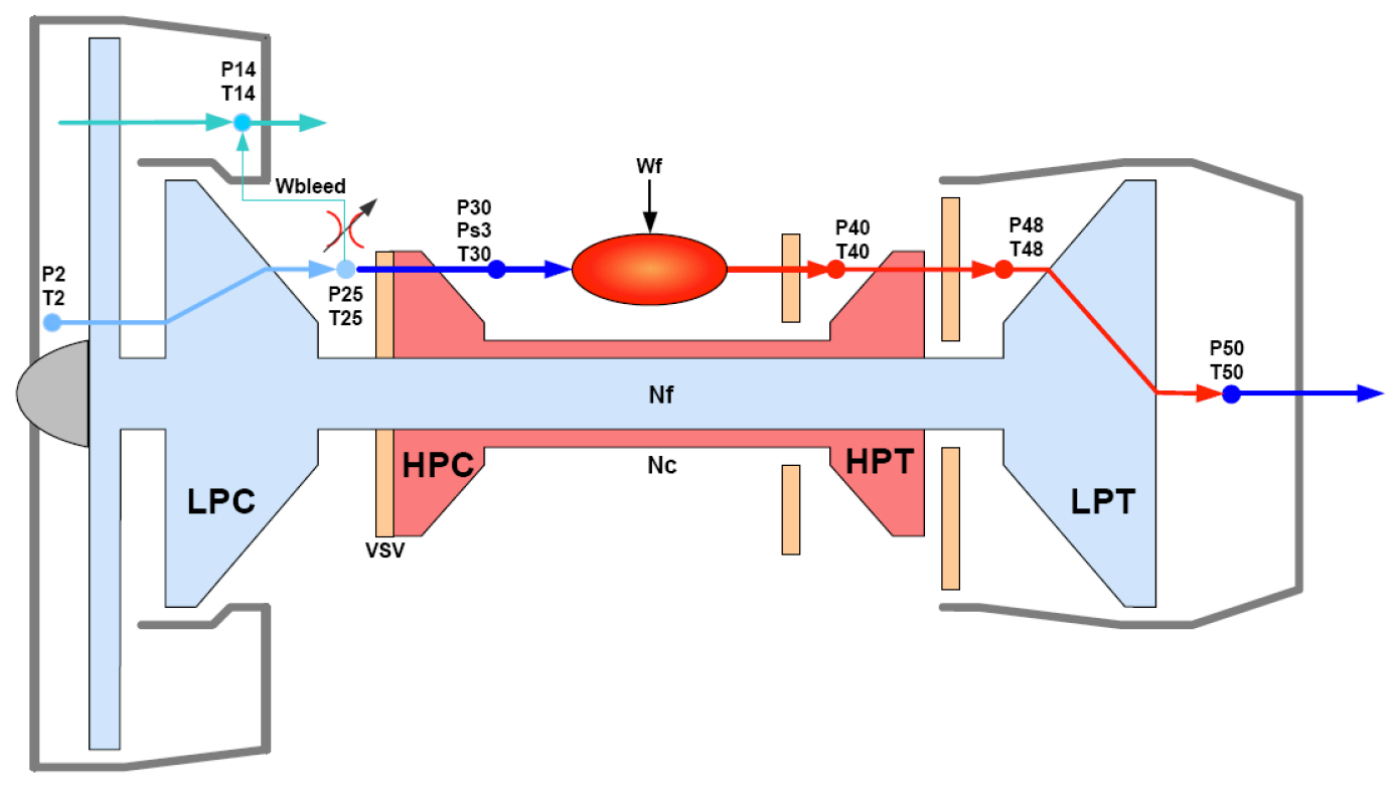

Figure 4. Schematic of CMAPSS40k engine.

\section{B. Engine Parameter Estimation}

One of the critical enabling technologies for MBEC is the improvement of accurate engine parameter estimation throughout the life cycle of the engine. On-board modeling of engine parameters, some of which currently need to be estimated, can be used in addition to sensed parameters as a means to improve control of the engine. ${ }^{17,18}$ One area of difficulty is maintaining estimation accuracy over the engine life cycle due to the limited sensors and the multitude of health parameters impacting the degradation of the engine. While various filtering techniques ${ }^{19-21}$ have been used in simulation in the past, the approach selected here is the $\mathrm{OTKF}^{22,23}$ to directly address the underdetermined problem. A linear and nonlinear implementation of the optimal tuner approach implemented in this paper is discussed below.

\section{Linear Optimal Tuner Estimation}

The OTKF provides updates to the on-board engine model, which enables the on-board model to selftune to account for engine performance variations. The estimation technique was successfully used in engine testing for integrated vehicle health management. ${ }^{24}$ For the optimal tuner estimation, the eight CMAPSS40k control sensors as listed above are used. A total of ten health parameters and eight sensors represents an underdetermined estimation problem, thus illustrating the need for the OTKF approach. The on-board model is a piecewise linear, time-invariant, state space model developed from CMAPSS40k at an altitude from 0 to 40,000 ft in steps of 20,000 ft, Mach number from 0 to 0.8 in steps of 0.2 , and corrected fan speed from 1200 to $4200 \mathrm{rpm}$ in steps of $400 \mathrm{rpm}$. The piecewise linear model is used to establish the Kalman Filter estimation updates based on sensor inputs. The estimated parameters for this study are the thrust, HPC SM, and T40.

For completeness, the main equations for the development of the OTKF are presented in the Appendix. The state vector, $x$, consists of speeds of the fan and core spools. The control input, $u$, represents the fuel flow and other control inputs such as, VSV and VBV. The measured output, $y$, represents all of the sensed parameters in the system. The parameter, $z$, represents the values of unmeasured parameters of interest. The vector, $h$, represents the health parameters of the engine. The estimation accuracy of the Kalman filter is dependent on the selection of $V^{*}$, which represents the transformation matrix relating health and tuning parameters discussed further in the Appendix. To make this selection, an optimal iterative search is used to minimize the mean sum of squared estimation errors in the parameters of interest, such as health parameters and auxiliary parameters that are not measured with sensors. ${ }^{25}$ 


\section{Nonlinear Optimal Tuner Estimation}

The OTKF was further developed to account for nonlinearities by implementing an optimal tuner extended Kalman filter (OTEKF) that can improve transient estimation. ${ }^{26}$ Typical extended Kalman filters rely on partial derivative matrices calculated in real-time from the nonlinear model. This is done in order to calculate the Kalman gain at the current operating point during the simulation. However, even with the increased computing power of modern processors, there are still challenges associated with linearizing the nonlinear engine models in real-time, which include the ability to ensure that the engine model converges stably at every operating point. To avoid these practical issues associated with on-board linearization, a time-invariant Kalman filtering approach was implemented with the OTEKF. This implementation has the advantage that the nonlinear model is only required to be executed once per time step. The equations for a discrete OTEKF implementation are illustrated in the Appendix. ${ }^{26}$ For the extended Kalman filter implementation, the nonlinear engine model is executed assuming a fixed $50 \%$ deteriorated engine and does not require the user to calculate a globally optimal transformation matrix.

\section{Controller and Operability Margins}

The aircraft turbofan engine has to operate over a wide range of environmental conditions. The fact that the engine behaves differently at different environmental conditions adds to the complexity of the control design. One way of handling this complexity is to use gain scheduling, which takes advantage of interpolation in order to transition between conditions. A block diagram of the CMAPSS40k setpoint controller is shown in Fig. 5, where the primary control loop is closed on EPR for the baseline and

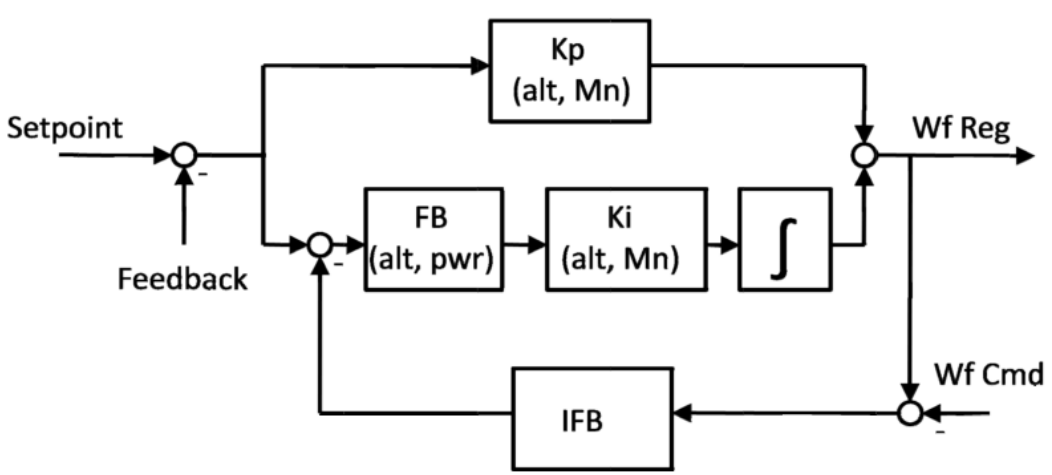

Figure 5. Gain scheduled setpoint controller block diagram. thrust for MBEC. The gains Kp and Ki of the PI controller used in CMAPSS40k are scheduled based on altitude and Mach number. ${ }^{2}$ There is an additional gain before the integrator that is scheduled based on the altitude and the power level (pwr). This aids in producing a critically damped response at different power levels. The integrator term contains an integral anti-wind-up protection scheme ${ }^{27}$ that includes the gain (IFB) as shown in Fig. 5. The output of the controller, Wf Reg, is the controller's desired fuel flow rate and $\mathrm{Wf} \mathrm{Cmd}$ is the actual fuel flow rate after the min/max protection logic at the last time step. For CMAPSS40k, the controller was designed to achieve the typical bandwidth of the fuel pump actuator of 6 $\mathrm{Hz}$ with a constraint of producing a gain margin of at least $6 \mathrm{~dB}$ and a phase margin greater than 45 degrees. This was achieved while maintaining a critically damped closed-loop response for both the EPR and thrust implementations.

As previously stated, the limiters in the CMAPSS40k controller restrict shaft speeds, combustor pressure, engine acceleration, and minimum RU to maintain safe operation throughout the flight envelope. ${ }^{2}$ The protection logic is designed to ensure that critical variables do not exceed their limits. In CMAPSS40k, each limit regulator is implemented as a PI controller, similar to the setpoint controller shown in Fig. 5. The main difference here is that there is no gain (FB) and the PI gains are constant, not scheduled. The primary limiter discussed here

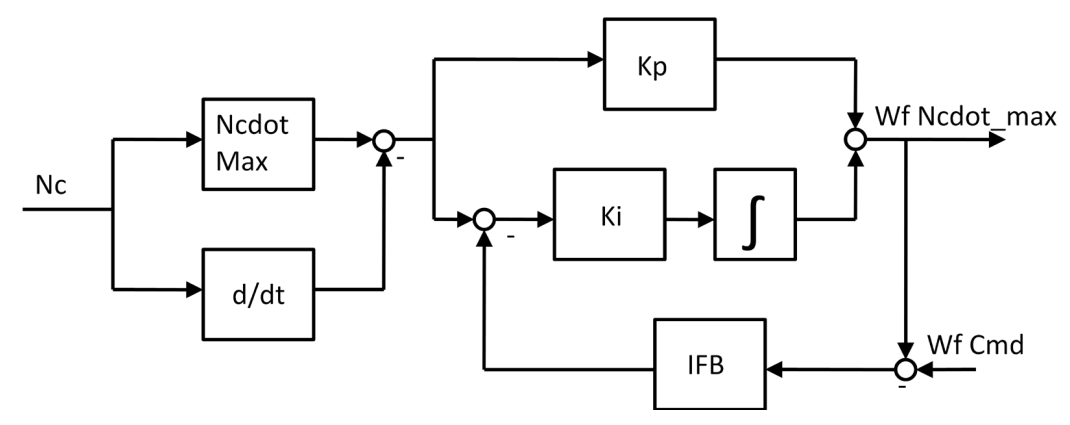

Figure 6. Diagram of acceleration limiter. 
is the acceleration limiter for the baseline, and similarly the HPC SM for MBEC. The purpose of these limiters is to prevent HPC stall during quick accelerations or large changes in thrust, since these maneuvers can cause a rapid acceleration. A diagram of the acceleration limiter structure is shown in Fig. 6, and is very similar to the general structure of all the other limiters used in this study. The acceleration schedule implemented in the baseline controller of C-MAPSS40k was designed using core acceleration (Ncdot) versus corrected core speed (Nc). Thus, during transients, the engine core is controlled to accelerate along a schedule that is a function of the current core speed. The max acceleration limit is determined from the acceleration schedule, shown in Fig. 6 as the Ncdot Max block. The error is computed from the acceleration max (Ncdot Max) and the current acceleration calculated from the current core speed. The primary difference in the HPC SM limiter is that instead of an Ncdot schedule, the limiter is based on a set SM.

\section{Potential Controller Enhancements}

In addition to the MBEC architecture, a conditionally active (CA) limiter was implemented to improve the performance of the above described protection logic. The CA method was developed ${ }^{28}$ wherein the engine control limit regulators become active only when the variable to be limited is within a specified bound that is close to the actual limit value. Consider the form of a Min-Max control selection scheme as shown in Fig. 2. The limit regulated outputs are constantly being used in the selection of the commanded input to the plant. Such an approach is inherently conservative since the commanded input might be limited by one of the limit regulators, even if the value of the output to be limited is far from its limit. Since the purpose of the limit regulator is to ensure that the specified limit is not violated, it does not need to be active if there is no chance of the limit being reached.

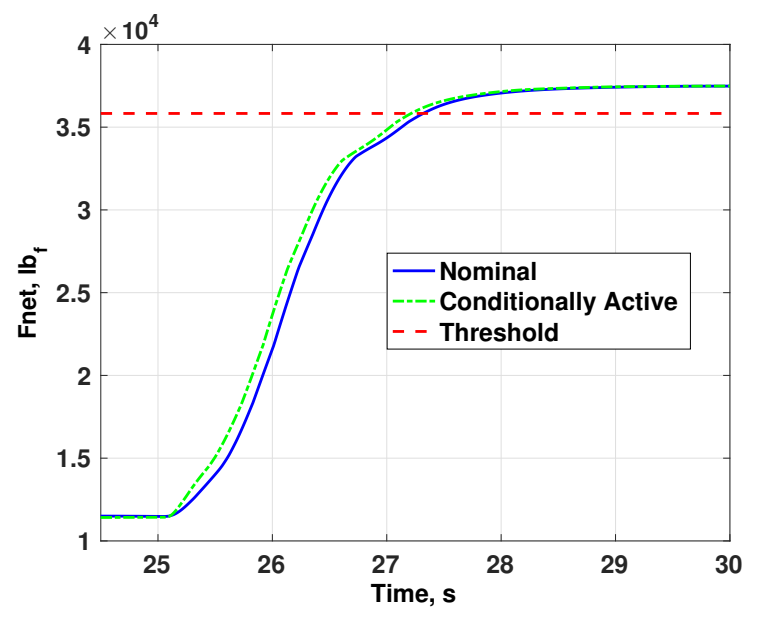

Figure 7. Conditionally active limiter improvement assuming perfect estimation.
The idea behind CA limit regulators is to make the limit regulator active in the Min-Max selection scheme only when there is a reasonable chance that the limit will be reached if the input command generated by the setpoint regulator (or another limit regulator) is not modified. ${ }^{28}$ An operational region for a limit regulator to be active in the Min-Max selection scheme can be defined by two conditions, which both need to be met. Condition one: the variable to be limited is close to its limit value; and condition two: its current rate is such that it will reach the limit within a certain period of time with no other changes. The use of conditionally active limit regulators enables faster engine response by preventing limit regulators from unnecessarily slowing the engine response. ${ }^{28}$ The CA limiter adds criteria that allows the limited variable to approach the actual limit much closer and only become active when the limit is in danger of being violated. Therefore, using the CA limit regulator logic and decreasing the acceleration limit can achieve fuel savings by decreasing the amount of required transient surge margin.

The CA control limiters are implemented with the MBEC architecture to reduce the rise time of the engine going from idle to full power to simulate a takeoff condition. Previous studies ${ }^{28}$ showed that the rise time could be reduced by over 0.5 seconds when compared against the baseline control architecture. However, with the HPC SM limiter, only a marginal improvement was obtained as illustrated in Fig. 7. The conditionally active HPC SM limiter provides a faster response (green), but it is only about $0.2 \mathrm{~s}$ faster from a sea level static idle to $95 \%$ power transient without a CA limiter (blue), even when assuming a perfect estimation. The CA limiter was used in the MBEC architecture, but its purpose is to provide additional margin to account for estimation errors instead of directly reducing the transient operability margins. 


\section{Application of MBEC to Engine Design}

MBEC has previously been explored for efficiency improvements in gas turbines by modifying the operating line of the engine. ${ }^{29-31}$ Initially, the control actuators such as VSV and VBV were investigated to modify operation of an already designed turbofan engine. However, it was determined that once a commercial engine is designed, the available actuators do not allow for significant changes to the steady state operating point of the engine. To fully realize the potential efficiency gains of MBEC, control considerations need to become part of the design process. This requires a paradigm shift to consider transient impacts during the design phase that have typically relied on steady state analyses. ${ }^{32}$ The approach to investigate efficiency improvements over baseline efficiency is the following:

\section{Steps for Controls in the Engine Design Process}

1. Operability Uncertainty Margin Investigation: Define the engine HPC SM stack up and identify potential reductions in operability margin.

2. MBEC Estimation Investigation: Develop MBEC controller and operability limit architecture to determine the estimation accuracy.

3. Operability Transient Margin Investigation: Define the minimum transient margin required to meet the FAA transient requirement.

4. Engine Redesign: An approximate engine redesign is conducted by modifying the turbine model flow scalars to allow for greater flexibility in setting the operating line with reduced HPC SM.

5. Tune the MBEC: The trim points and dynamics could change from the baseline engine due to the newly designed engine. To ensure the previously determined estimation accuracy, tune the OTKF.

6. Results for TSFC Reduction: Implement the MBEC architecture on the redesigned engine to realize efficiency improvements and ensure safe operation across the flight envelope.

7. Iteratively verify with engine designers that efficiency improvements are achieved.

Table 1. Typical stall margin uncertainty stack up used for operability limits.

\begin{tabular}{|l|c|}
\hline $\begin{array}{l}\text { Elements of Stall Margin } \\
\text { Uncertainty }\end{array}$ & $\%$ \\
\hline $\begin{array}{l}\text { Engine to Engine } \\
\text { Variation }\end{array}$ & $1.0 \%$ \\
\hline Reynolds Number Effects & $1.0 \%$ \\
\hline $\begin{array}{l}\text { Operating Line } \\
\text { Deterioration }\end{array}$ & $2.0 \%$ \\
\hline Stall Line Deterioration & $3.0 \%$ \\
\hline Inlet Distortion & $4.0 \%$ \\
\hline $\begin{array}{l}\text { Total Stall Margin } \\
\text { Uncertainty }\end{array}$ & $11 \%$ \\
\hline
\end{tabular}

components corresponding to deterioration.
These steps will be outlined for the application to CMAPSS40k in the following subsections. The one step not taken in this process for the current study is the iteration with the engine designers.

\section{A. Operability Uncertainty Margin Investigation}

The stall margin stack up is designed at the cruise operating point that accounts for various impacts that will cause the margin to fluctuate at various operating conditions throughout the engine's operating life such as engine-to-engine variation, deterioration, transient operations, and distortion. ${ }^{10,33,34}$ For the purposes of this study the SM will be broken down into two main categories of uncertainty margin and transient margin, as was shown in Fig. 1. In this section the focus is on the uncertainty margin that can be broken into elements shown in Table $1 .^{33}$ Some of the uncertainty stack shown in Table 1 . is fixed due to possible conditions that the engine is expected to experience over its operating envelope or life cycle. The portion of the stack up explored for reduction here are the two 
To determine the conservatism in the operability margin of the engine, a baseline cruise design point is defined at $30,000 \mathrm{ft}$ altitude, 0.8 Mach flight speed, and a power setting of 66.5 degrees PLA. The HPC SM at this design point was determined to be $24.05 \%$ for a new engine. The HPC SM stack up is shown in Fig. 8. For the transient component, the faster engine response is explored for reduction and shown in green. This will be discussed in the next two following subsections. For the uncertainty component, deterioration is explored for a possible reduction in the operability margin, highlighted in green. The values in the highlighted green reduction blocks for the operability margin provided in Fig. 8 are obtained through simulation analysis with CMAPSS40k.

The conservatism in the HPC SM stack is then investigated by simulating 2557 flight conditions run across the flight envelope and engine life cycle to find variations from the defined cruise design point. For the uncertainty stack, the steady-state mid-life $(50 \%$ deteriorated engine) worst case SM was $11.11 \%$, an average of $14.28 \%$, and three standard deviations of $3.96 \%$. Uncertainty in the HPC SM component due to engine life deterioration had a worst case of $5.01 \%$, an average of $2.37 \%$, and three standard deviations of $0.80 \%$. This leaves approximately $13 \%$ margin for transient operation from the already mentioned $24 \%$. The trade study illustrated that the uncertainty in SM due to deterioration could be reduced by $1.8 \% \mathrm{SM}$ if the margin was set based on the average value plus three standard deviations instead of the worst case of $5.01 \%$. This is illustrated as a green block in the modified stack up in Fig. 8. This establishes the uncertainty component for reducing the HPC SM for engine redesign with an operating line closer to the stall line.

\section{B. MBEC Estimation Investigation}

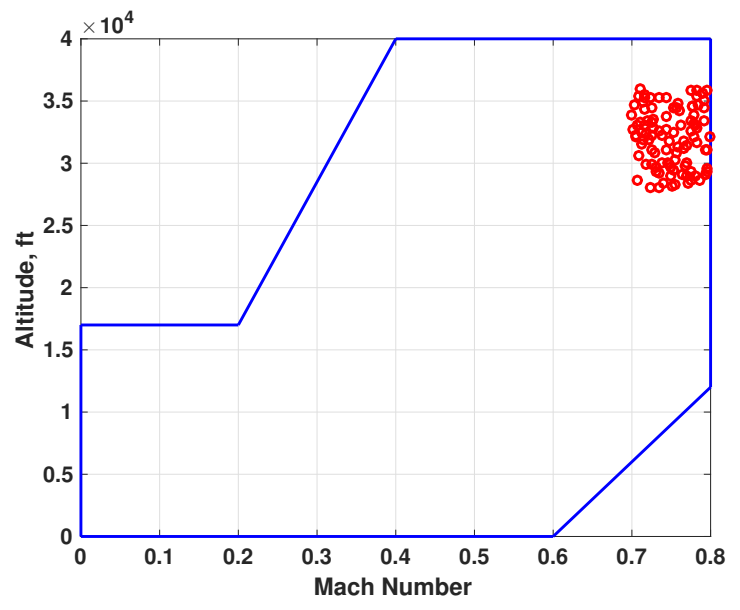

Figure 9. Cruise flight envelope for CMAPSS40k.

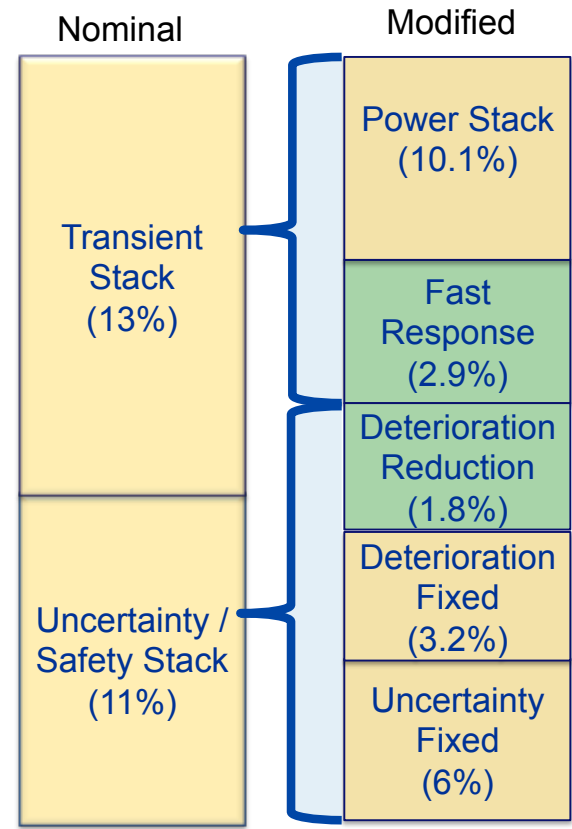

Figure 8. Stall margin stack up used for operability limits in designing limiters and operating schedules. The further breakdown of the uncertainty and transient components are used to help illustrate the potential elements of reduction shown in green.

was run with a 10 degree PLA step up and down to capture both the steady and transient maximum percent difference. In Fig. 10, the left plot, (a), shows the steady state, and the right plot, (b), shows the transient percent difference. It should be noted that the HPC SM is shown as a relative percent error and not the absolute value. The general trend is that the error increases during the transient maneuvers. In steady state the HPC SM difference peaks at only about $1 \%$, while in the transient there were cases that reached approximately $11 \%$. The estimation accuracy is sufficient to reduce the HPC SM stack up for the CMAPSS40k 
operating line; however, some additional margin was added to account for the HPC SM estimation error.
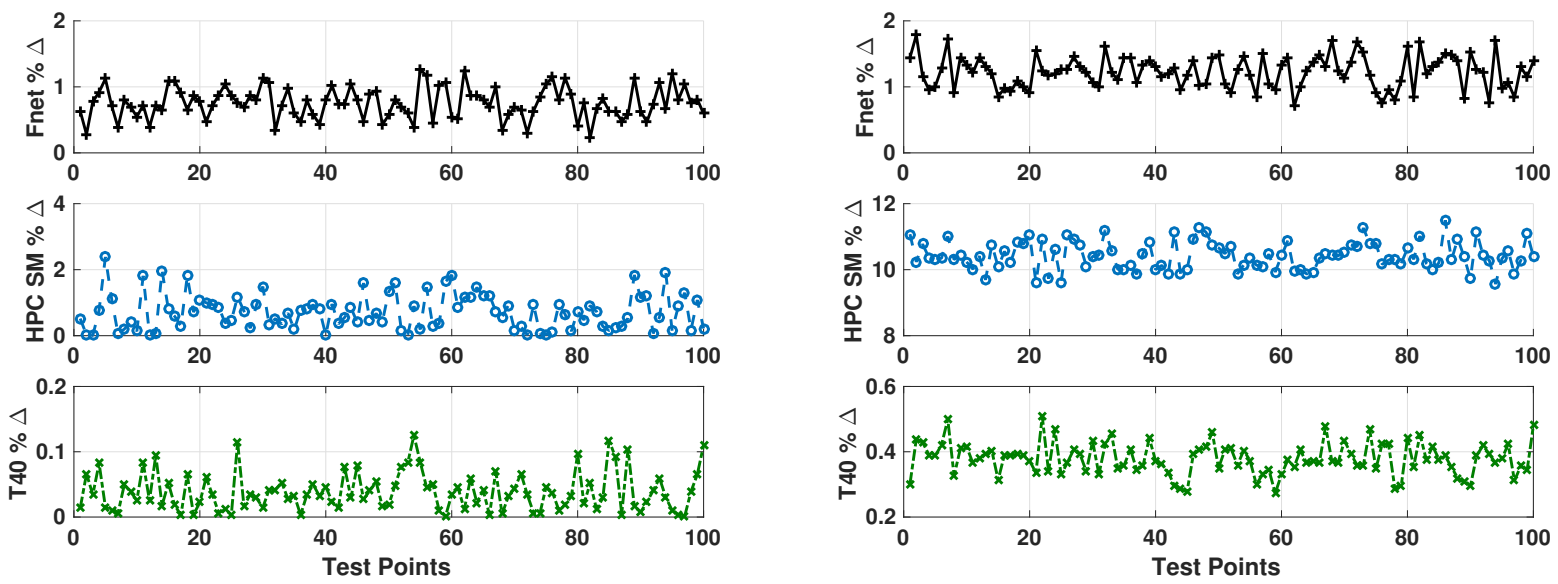

(a) Steady-State

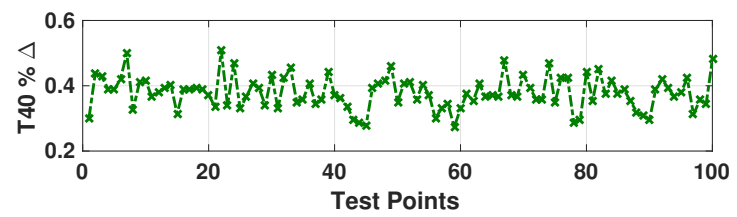

(b) Transient

Figure 10. Comparison of the true and estimated values of thrust, HPC SM, and T40 at steady state (a) and transient (b) using the OTKF. The maximum percent difference is shown across the hundred operating points, note the HPC SM is the relative percent error.
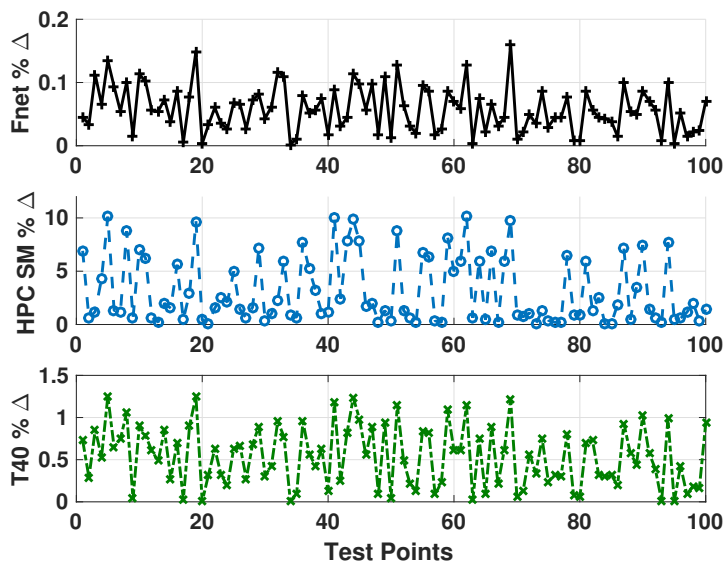

Figure 11. Comparison of the true and estimated values of thrust, HPC SM, and T40 during transient operation using the OTEKF. The maximum percent difference is shown across the hundred operating points, note the HPC SM is the relative percent error.

In order to improve the estimation of the engine parameters, particularly during transient maneuvers, an extended Kalman filter was implemented. The optimal tuner extended Kalman filter (OTEKF) ${ }^{26}$ is run at the same one hundred cruise operating points to compare against the linear OTKF. Note that there are no changes to the controller's architecture, only changes to the on-board model Kalman filter. The OTEKF relies on the use of a nonlinear engine model to produce the output vector, and auxiliary state vector which contains the estimated parameters for the controller. The maximum percent difference for the three engine parameters during transient operation is shown in Fig. 11. This shows the advantage of implementing the OTEKF when compared to the OTKF in Fig. 10. A significant reduction in the estimation difference for the thrust and the mean estimation difference for the HPC SM is shown, while the estimation difference for T40 still remains relatively small. The key parameters for this study are the estimation of thrust and HPC SM. This indicates that significantly less margin is required to account for estimation errors if the mean error from OTEKF is used, or at least a marginal reduction if the worst case is used. For the purposes of this study it is assumed that the mean estimation error from the OTEKF is sufficient. 


\section{Operability Transient Margin Investigation}

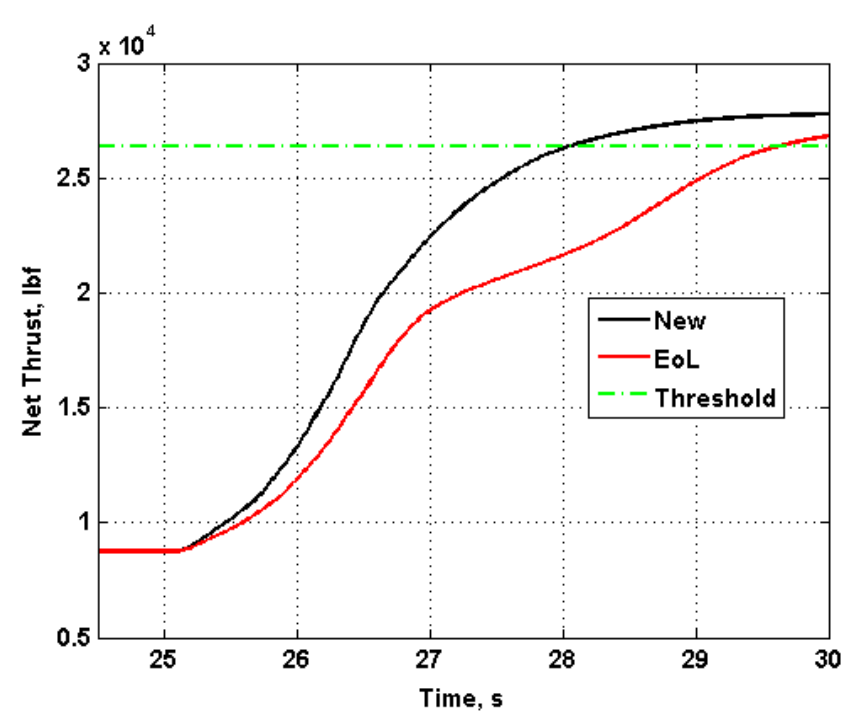

Figure 12. Reduction in transient margin by iterating to meet the FAA requirement with minimum required margin.
In addition to the uncertainty portion of the HPC SM, there is a potential reduction in the transient margin. ${ }^{32}$ This is possible based on the engine response adequately meeting Federal Aviation Administration (FAA) transient takeoff requirement, ${ }^{35}$ which is defined as going from flight idle to $95 \%$ power within five seconds. Takeoff simulations are used to determine additional reduction of the $13 \%$ transient margin for the FAA transient performance requirement. To test this with the engine model, the MBEC stall margin limiter is used to set the transient stack, assuming perfect estimation. A worst case scenario is used to account for potential estimation error by lowering the available HPC SM at takeoff due to running the simulation at an altitude of $8000 \mathrm{ft}$ and on a hot day $\left(50^{\circ} \mathrm{R}\right.$ over the standard day). The simulation is setup for the large transient to occur at 25 seconds to ensure a steady state condition

is met, and then run to $95 \%$ of full power for the new engine. The transient margin is reduced until the EoL engine meets the FAA transient requirement with a small delta margin to account for estimation errors in the final MBEC architecture. In the baseline CMAPSS40k the engine can meet the requirement in a couple seconds, but as the faster response operability margin is reduced, the response of the engine slows down. This reduced time response is shown in Fig. 12, for both a new and EoL engine. It can be seen that the EoL engine still meets the FAA transient requirement with a little less than a half second additional response time to spare. This resulted in a transient reduction in the operability margin of $2.9 \%$, making the total SM reduction to be $4.7 \%$.

\section{Engine Redesign}

An approximate redesign of this engine is conducted by moving the operating line, given a reduction in the operability margin. The redesign is accomplished by scaling the flow scalars of the LPT and HPT performance maps to reduce the corrected mass flow, thus altering the engine operating line to a higher pressure ratio. This is done to illustrate the potential added flexibility and efficiency gains provided to an engine designer if controls are considered during the design phase. A wider range of a compressor performance map can be used for operation due to MEBC, for its ability to meet performance operability requirements with reduced margins.

A couple percent reduction in the flow scalars starts an iterative search approach, until the operating line conservatism is reduced, to account for the $4.7 \%$ margin identified as the desired reduction. The final flow scalars chosen for the engine redesign were $94.75 \%$ of the initial corrected values for both the LPT and HPT. This essentially accounts for the transient reduction, but trades the uncertainty margin with the estimation error. The original and modified CMAPSS40k operating line for the HPC and HPT are shown in Fig. 13. The turbine redesign alters the operating line of the compressive system components, moving them towards the stall line (yellow). The new operating line for the HPC (green) is closer to the stall line (yellow) than the original operating line (blue). Here the turbine is operating in a choked or near choked condition, and the operating region is small compared to the compressive system components. These small changes in the turbine, then, have large impacts on the compressive system components. The color map illustrates the efficiency, with red indicating higher efficiency and blue indicating lower efficiency. For the OTEKF, the flow scalars of $95.75 \%$ are used, instead of the flow scalars of $94.75 \%$ for the OTKF. This accounts for most of the uncertainty reduction, due to the greater confidence in the estimation accuracy during transients for the OTEKF. 

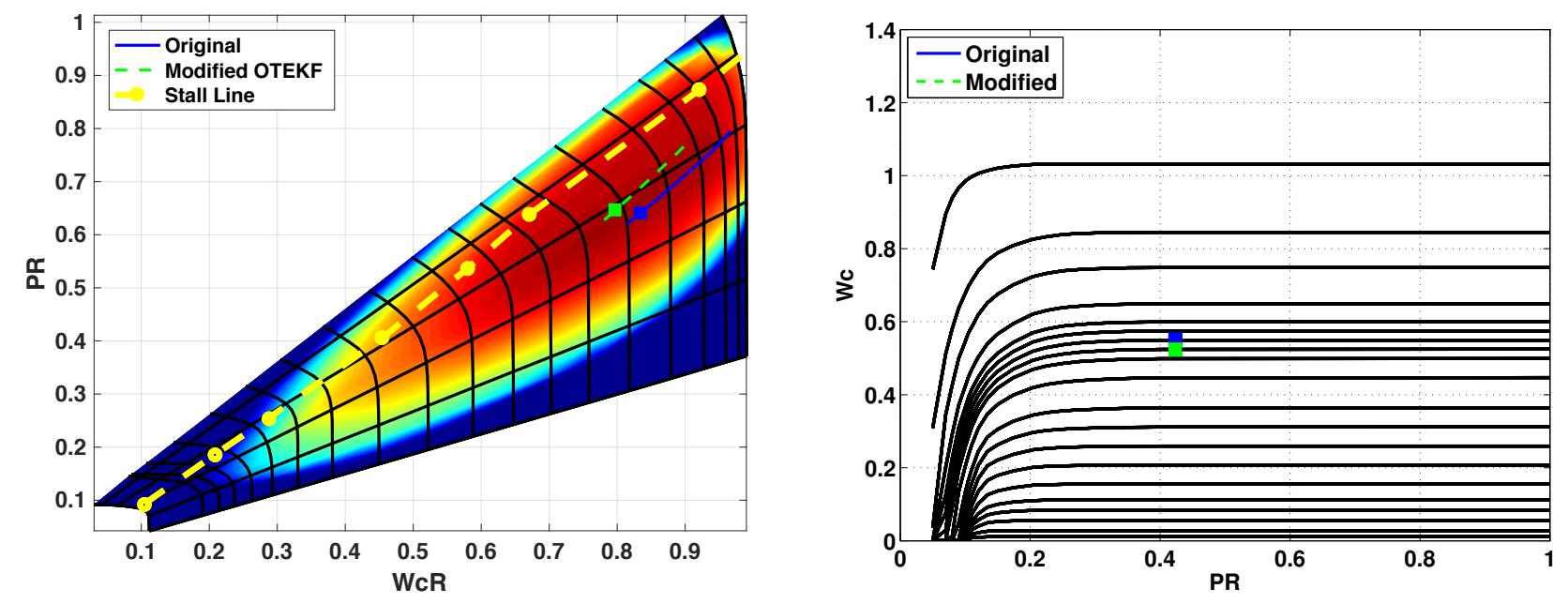

Figure 13. Performance maps of HPC, left, and HPT, right, with the baseline engine (original) and redesigned engine (modified) operating line. The change in the operating line that is shown is for the $94.75 \%$ flow scalars used in the OTEKF impelentation.

\section{E. Tune the MBEC OTKF}

The MBEC OTKF architecture is built upon a piece-wise linear model that is obtained from linearizing the CMAPSS40k simulation through small perturbations about an operating point. The linear point models are structured together through interpolation schemes and steady state trim points from the steady state flow balance solver. The redesigned engine from the previous subsection will have trim points and dynamics that are slightly off from the baseline engine used in the initial estimation accuracy analysis. To ensure that the same approximate estimation accuracy is achieved, a new piece-wise linear model is obtained for the redesigned engine. The same procedure of building the OTKF is then applied. In general, just by updating the trim points the majority of the estimation error between the baseline and redesign engine can be resolved. However, to ensure the same level of estimation accuracy shown in subsection IV, B: MBEC estimation investigation, the OTKF was tuned to the new model for the results that follow. The OTEKF is tuned to modify the Kalman gains; however, since it is applied to the nonlinear model the trim point updates are not required to be updated.

The final step of including controls in the engine design process illustrates the potential for TSFC reduction in the redesigned engine while maintaining safe operation. This step will be discussed in more detail in the following section.

\section{Results}

The following results show the efficiency improvements from the implementation of the MBEC architecture on the newly designed engine, compared to the CMAPSS40k baseline engine simulation with an EPR fuel flow controller and acceleration limiter. Results are first shown to illustrate that the newly designed engine, with an OTKF MBEC architecture, meets safe operability requirements. The FAA transient requirement to go from flight idle to $95 \%$ of full power is shown in Fig. 14. Here only the "true" values from CMAPSS40k are shown for both the thrust and HPC SM instead of estimates from the on-board model. A stall margin limit of $11 \%$ is used based on the operability analysis previously discussed. This illustrates that the MBEC is able to provide a relatively tight control of the SM, and there is only a slight violation of the limit due to estimation inaccuracies for the end of life engine. The end of life engine thrust response is able to easily make the $95 \%$ threshold within five seconds. The ability of the redesigned engine to meet the requirement is in part due to the simulation being run at sea level standard condition as required by the FAA, instead of the intentionally harsh takeoff condition that is used to set the margin reduction.

To compare the two engines at the one hundred cruise operating conditions, the thrust output from each baseline engine simulation, with an EPR controller, is used to set the thrust demanded for the corresponding 

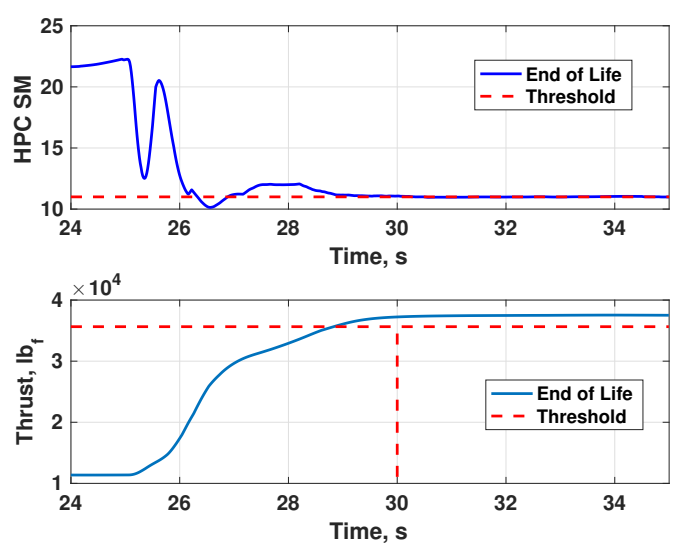

Figure 14. Transient margin reduction check.
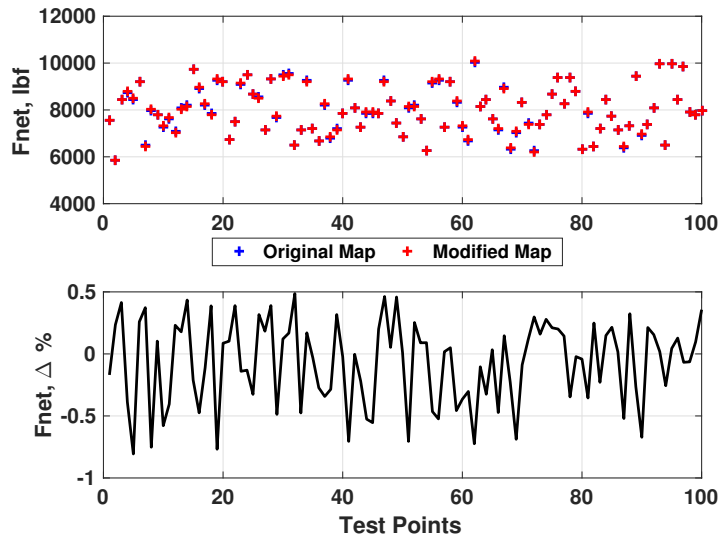

Figure 15. Steady state thrust matching between baseline and modified CMAPSS40k.

MBEC thrust controller simulation run. The thrust generated from the baseline engine (blue) closely matches that of the MBEC architecture with the redesigned engine (red), as shown in Fig. 15. For the cruise operating points the thrust variation is typically less than about $0.5 \%$ difference at steady state. This result also demonstrates that the redesigned engine with a reduced mass flow rate produces the same thrust as the baseline engine.

To ensure that the HPC SM is not violated in the redesigned MBEC engine architecture, its performance is compared to the SM limit set in this design, and also compared against the baseline CMAPSS40k. For the one hundred cruise operating points, the "true" minimum HPC SM from CMAPSS40k is shown in Fig. 16 for both the baseline CMAPSS40k (blue) and redesigned (red). The redesigned MBEC engine can clearly be seen to have less margin, as was expected in the design. The new HPC SM limiter for the MBEC architecture used a threshold of $11 \%$ HPC SM, and is shown as a red dashed line in Fig. 16. It can be seen that while the redesigned engine operates at a lower HPC $\mathrm{SM}$, the limiter allows for confidence that a defined level of safety is maintained. There are only very small violations of

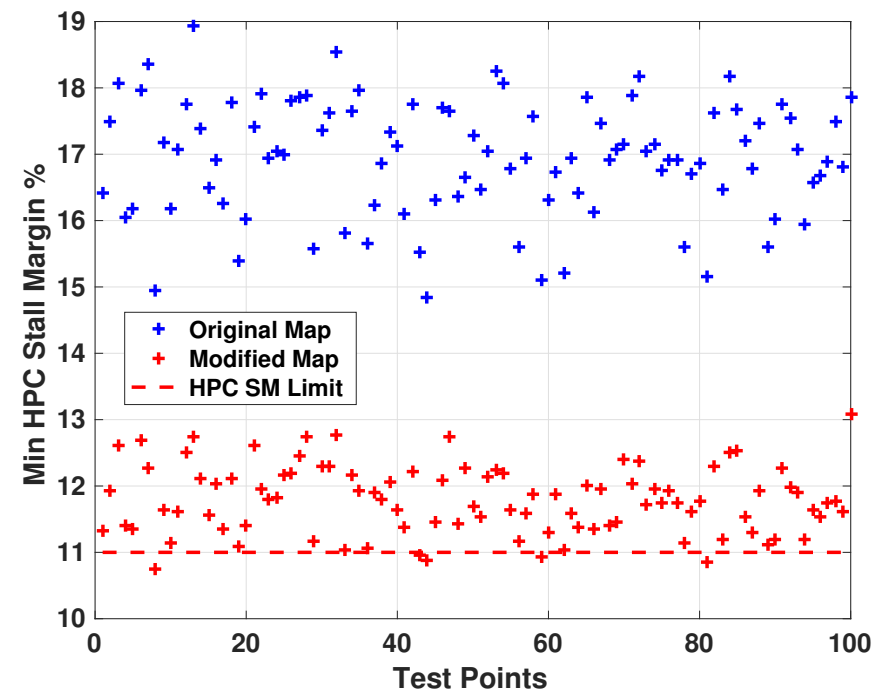

Figure 16. MBEC stall margin limiter results. the threshold due to the estimation errors in the OTKF implementation.

Given that the MBEC architecture is able to maintain safe operation of the redesigned engine, it can now be explored if the MBEC architecture can improve efficiency. A comparison of the steady state TSFC values between the baseline engine (blue plus) and redesigned engine with the OTKF implementation (red circle) is shown in the top plot of Fig. 17 (a). While the values are similar as shown in the top plot, the bottom plot of Fig. 17 (a) clearly shows the percent reduction in TSFC when compared to the baseline CMAPSS40k simulation for the one hundred cruise test cases. The MBEC architecture applied to the redesigned engine with estimation errors, sensor noise, and various deterioration levels still provides an average of $1.01 \%$ reduction in TSFC with the modest change to the operating line previously described.

To investigate potential further improvements in the TSFC reduction, the OTEKF implementation is 

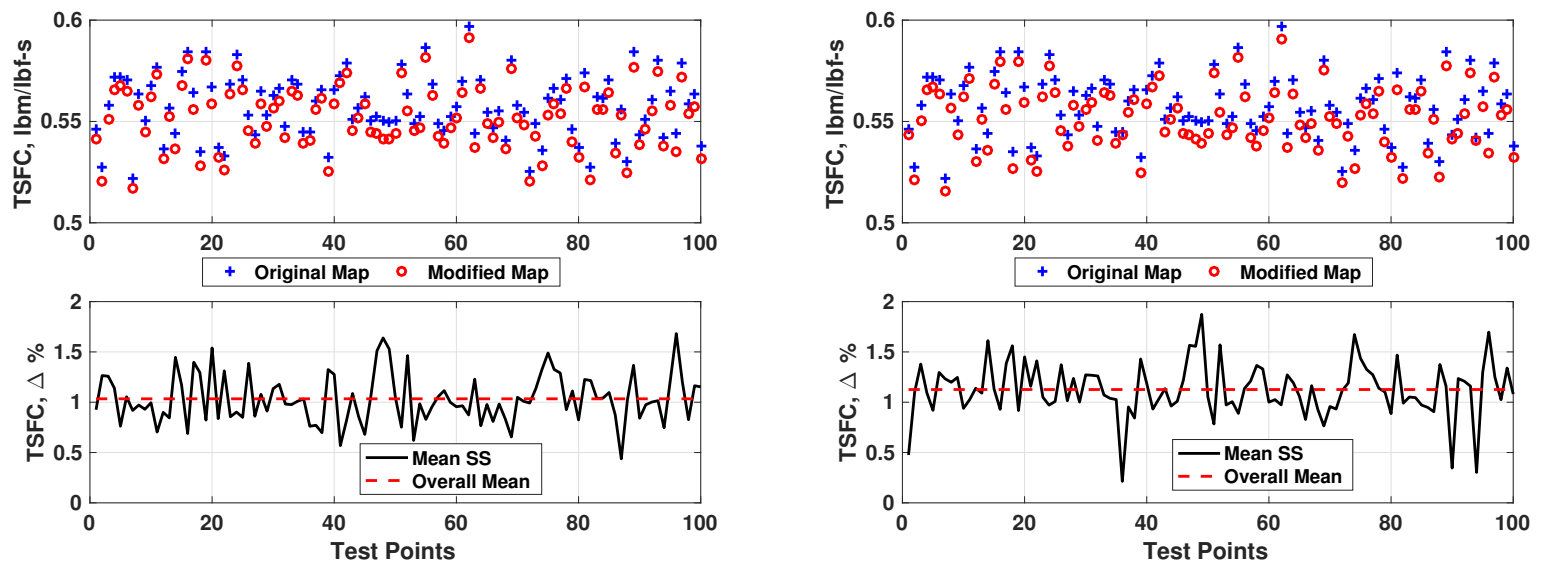

(a) OTKF

(b) OTEKF

Figure 17. TSFC generated from the baseline map and EPR controller compared to the MBEC architecture with the modified maps and percent improvement of TSFC.

shown in Fig. 17 (b). Further improvement is obtained by the improved estimation accuracy during transient maneuvers due to the OTEKF. This allows for the larger turbine flow scalars to be used to fully take advantage of potential operability margin reduction. The improved estimation allows for the reduction in the HPC SM limiter to be set to $9 \%$, instead of the $11 \%$ used for the OTKF implementation. The same safe operation is investigated with the lower limit value, as was shown in Fig. 16. The additional operability margin reduction with the OTEKF is shown to have an approximate $10 \%$ improvement in the TSFC reduction to $1.12 \%$. This work has illustrated how advanced controls can impact the engine design and, with modest changes to the operating line, can provide a reduction in TSFC during steady-state cruise operation.

\section{Conclusions}

The application of a model-based engine control (MBEC) design, applied to the Commercial Modular Aero-Propulsion System Simulation 40,000 (CMAPSS40k) turbofan engine simulation with an optimal tuner Kalman Filter estimation routine for the on-board model, was shown. MBEC is used to develop a methodology to show how advanced control technologies can be used to improve efficiency, during the design phase of a turbofan engine, by reducing conservative operability margins. MBEC enables a tighter control of operability margins, which can enable reductions of the stall margin, thereby expanding the engine design space and potential for efficiency improvements. In addition to the traditional Kalman filter, an optimal tuner extended Kalman Filter was investigated to improve the on-board model estimates during transients for a tighter control of the operability margins. A redesigned engine is approximated by modifying the turbine flow scalars to reduce the compressor stall margin. The MBEC architecture implemented on the redesigned engine was compared to the CMAPSS40k baseline simulation and showed a thrust specific fuel consumption reduction of $1.12 \%$. In practice, these improvements will need to be verified with engine system designers.

\section{Appendix}

\section{A. Optimal Tuner Kalman Filter}

The overall engine plant model is described by a linear time-invariant state space system described in Eq. 1 to Eq. 3. To establish the Kalman Filter, uncorrelated zero-mean white noise is introduced represented by $w$ and $v$, which then establishes the covariance matrices $Q$ and $R$ respectively. The system matrices of $A, B, C, D, L, M, F, G$, and $N$ are of appropriate size to establish the system. 


$$
\begin{aligned}
\dot{x} & =A x+B u+L h+w \\
y & =C x+D u+M h+v \\
z & =F x+G u+N h
\end{aligned}
$$

To account for how the health parameters induce shifts in the engine state variables, the health parameter vector can be directly concatenated with the state vector. The health parameters are modeled without dynamics since the gradual deterioration over the life of an engine is much slower than the other state variables. In an augmented form, the system is now shown in Eq. 4 to Eq. 6, where the subscript " $x h$ " denotes the combined state and health vectors.

$$
\begin{aligned}
\dot{x}_{x h}=\left[\begin{array}{c}
\dot{x} \\
\dot{h}
\end{array}\right] & =\underbrace{\left[\begin{array}{cc}
A & L \\
0 & 0
\end{array}\right]}_{A_{x h}} \underbrace{\left[\begin{array}{c}
x \\
h
\end{array}\right]}_{x_{x h}}+\underbrace{\left[\begin{array}{c}
B \\
0
\end{array}\right]}_{B_{x h}} u+\underbrace{\left[\begin{array}{c}
w \\
w_{h}
\end{array}\right]}_{w_{x h}} \\
& =A_{x h} x_{x h}+B_{x h} u+w_{x h} \\
y & =\underbrace{\left[\begin{array}{ll}
C & M
\end{array}\right]}_{C_{x h}} \underbrace{\left[\begin{array}{l}
x \\
h
\end{array}\right]}_{x_{x h}}+D u+v \\
& =C_{x h} x_{x h}+D u+v \\
z & =\underbrace{\left[\begin{array}{ll}
F & N
\end{array}\right]}_{F_{x h}} \underbrace{\left[\begin{array}{l}
x \\
h
\end{array}\right]}_{x_{x h}}+G u \\
& =F_{x h} x_{x h}+G u
\end{aligned}
$$

To account for the health parameter underdetermined estimation problem, a tuning vector is used defined by Eq. 7 .

$$
q=V^{*} h
$$

Here, $V^{*}$ is a transformation matrix that is applied to construct the tuning vector. A pseudo-inverse of the transformation matrix, $V^{* \dagger}$, is used to obtain an approximation of the health parameter vector.

$$
\hat{h}=V^{* \dagger} q
$$

More details of the optimal tuner approach can be found in Simon, ${ }^{23,25}$ where the optimization of $V^{*}$ is developed. This relationship between the health parameters and tuners leads to the following reduced order system: 


$$
\begin{aligned}
\dot{x}_{x q}=\left[\begin{array}{c}
\dot{x} \\
\dot{q}
\end{array}\right] & =\underbrace{\left[\begin{array}{cc}
A & L V^{* \dagger} \\
0 & 0
\end{array}\right]}_{A_{x q}} \underbrace{\left[\begin{array}{c}
x \\
q
\end{array}\right]}_{x_{x q}}+\underbrace{\left[\begin{array}{c}
B \\
0
\end{array}\right]}_{B_{x q}} u+\underbrace{\left[\begin{array}{c}
w \\
w_{q}
\end{array}\right]}_{w_{x q}} \\
& =A_{x q} x_{x q}+B_{x q} u+w_{x q} \\
y & =\underbrace{\left[\begin{array}{ll}
C & M V^{* \dagger}
\end{array}\right]}_{C_{x q}} \underbrace{\left[\begin{array}{l}
x \\
q
\end{array}\right]}_{x_{x q}}+D u+v \\
& =C_{x q} x_{x q}+D u+v \\
z & =\underbrace{\left[\begin{array}{ll}
F & N V^{* \dagger}
\end{array}\right]}_{F_{x q}} \underbrace{\left[\begin{array}{l}
x \\
q
\end{array}\right]}_{x_{x q}}+G u \\
& =F_{x q} x_{x q}+G u
\end{aligned}
$$

The Kalman Filter is developed using a steady state approach, where the state estimation covariance and the Kalman gain do not change in time. By solving the Ricatti equation in Eq. 12 for $P$, the Kalman gain can be obtained in Eq. 13. An estimation of the reduced order state vector is then obtained by Eq. 14 .

$$
\begin{aligned}
0 & =-P C_{x q}^{T} R^{-1} C_{x q} P+A_{x q} P+P A_{x q}^{T}+Q_{x q} \\
K & =P C_{x q}^{T} R^{-1} \\
\dot{\hat{x}}_{x q} & =A_{x q} \hat{x}_{x q}+B_{x q} u+K\left(y-C_{x q} \hat{x}_{x q}-D u\right) \\
& =\left(A_{x q}-K C_{x q}\right) \hat{x}_{x q}+K y+\left(B_{x q}-K D\right) u
\end{aligned}
$$

The controller is defined by Eq. 15 to Eq. 19

$$
\begin{aligned}
\dot{x}_{c} & =A_{c} x_{c}+B_{c e} e+B_{c o} y_{o} \\
u & =C_{c} x_{c} \\
e & =r-\hat{z}_{r} \\
\hat{z}_{r} & =\left[\begin{array}{ll}
F_{r} & N_{r} V^{* \dagger}
\end{array}\right] \hat{x}_{x q}+G_{r} u \\
y_{o} & =C_{o} x+D_{o} u+M_{o} h
\end{aligned}
$$

Combining the above controller relationships with the Kalman Filter estimate gives Eq. 20.

$$
\begin{aligned}
\dot{x}_{c} & =\left(B_{c o} C_{o}\right) x+\left(A_{c}-B_{c e} G_{r} C_{c}+B_{c o} D_{o} C_{c}\right) x_{c}-B_{c e}\left[\begin{array}{ll}
F_{r} & N_{r} V^{* \dagger}
\end{array}\right] \hat{x}_{x q}+B_{c o} M_{o} h+B_{c e} r \\
& =A_{1} x+A_{2} x_{c}+A_{3} \hat{x}_{x q}+L_{1} h+B_{c e} r
\end{aligned}
$$

Finally, combining the augmented plant with the Kalman Filter and the controller, the closed loop system can be written as:

$$
\left[\begin{array}{c}
\dot{x} \\
\dot{x}_{c} \\
\dot{x}_{x q} \\
\dot{h}
\end{array}\right]=\left[\begin{array}{cccc}
A & B C_{c} & 0 & L \\
A_{1} & A_{2} & A_{3} & L_{1} \\
K C & B_{x q} C_{c} & \left(A_{x q}-K C_{x q}\right) & K M \\
0 & 0 & 0 & 0
\end{array}\right]\left[\begin{array}{c}
x \\
x_{c} \\
\hat{x}_{x q} \\
h
\end{array}\right]+\left[\begin{array}{c}
0 \\
B_{c e} \\
0 \\
0
\end{array}\right] r
$$




\section{B. Optimal Tuner Extended Kalman Filter}

A discrete extended Kalman filter implementation is illustrated below. ${ }^{26}$ Let the a priori estimate be equal to the previous state estimate:

$$
\hat{x}_{k}^{-}=\hat{x}_{(k-1)^{+}}
$$

Therefore, the a posteriori state estimation equation, can be written as:

$$
\hat{x}_{k}^{+}=\hat{x}_{k}^{-}+K_{\infty}\left(y_{k}-\hat{y}_{k}^{-}\right)
$$

where ideally:

$$
\hat{y}_{k}^{-}=C_{x q} \hat{x}_{k}^{-}+D_{x q} \Delta u
$$

This equation shows that $\hat{y}_{k}^{-}$is a function of the a posteriori estimate, $\hat{x}_{k}^{-}$, which is based on the previous estimate. Therefore, in a discrete implementation equation Eq. 23 can be written and implemented as:

$$
\hat{x}_{k}^{+}=\hat{x}_{k}^{-}+K_{\infty}\left(y_{k-1}-\hat{y}_{k-1}^{-}\right)
$$

The update portion of this equation, $K_{\infty}\left(y_{k-1}-\hat{y}_{k-1}^{-}\right)$, commonly known as the innovations, is based on the previous output of the nonlinear model and the sensed measurements. Once the estimated apriori state is updated, the nonlinear model is executed based on the current actuator commands, $u_{k}$, and current a posteriori estimate, $\hat{x}_{k}^{+}$, to produce the current output vector, $\hat{y}_{k}$, and auxiliary output vector $\hat{z}_{k}$ :

$$
\hat{y}_{k}=f\left(\hat{x}_{k}^{+}, u\right) \hat{z}_{k}=f\left(\hat{x}_{k}^{+}, u\right)
$$

\section{Acknowledgements}

The authors would like to acknowledge Don Simon for sharing his expertise in the optimal tuner Kalman Filter, Sanjay Garg and Jonathan Seidel for guidance in the development approach for investigating control impacts during engine design, and the Transformational, Tools, and Technologies project for their support of this research.

\section{References}

${ }^{1}$ Spang, H. A. and Brown, H., "Control of Jet Engines," Control Engineering Practice, Vol. 7, No. 9, 1999, pp. $1043-1059$.

${ }^{2}$ Csank, J., Ryan, M., Litt, J. S., and Guo, T., "Control Design for a Generic Commercial Aircraft Engine," NASA/TM 2010-216811, 2010.

${ }^{3}$ Greitzer, E. M., "Review - Axial Compressor Stall Phenomena," Journal of Fluids Engineering, Vol. 102, June 1980, pp. $134-151$.

${ }^{4}$ Adibhatla, S., Brown, H., and Gastineau, Z. D., "Intelligent Engine Control (IEC)," AIAA 1992-3484, 1992.

${ }^{5}$ Adibhatla, S. and Lewis, T. J., "Model-Based Intelligent Digital Engine Control (MoBIDEC)," AIAA 1997-3192, 1997.

${ }^{6}$ Kumar, K. K., Haschisako, Y., and Huang, Y., "Jet Engine Performance Estimation Using Intelligent System Technologies," AIAA 2001-1122, 2001.

${ }^{7}$ Litt, J. S., Simon, D. L., Garg, S., Guo, T.-H., Mercer, C., Millar, R., Behbahani, A., Bajwa, A., and Jensen, D. T., "A Survey of Intelligent Control and Health Management Technologies for Aircraft Propulsion Systems," Journal of Aerospace Computing, Information, and Communication, Vol. 1, 2004.

${ }^{8}$ Shin, J., "NASA Aeronautics: Strategic Implementation Plan," NASA NP 2015-03-1479, 2015.

${ }^{9}$ Carter, J. F., Tahmassebi, F., Chandler, F., Ginn, S., Gomez, R., Grabbe, S., Leavitt, L., Misra, A., Weathered, B., and Yamauchi, G., "NASA Technology Roadmaps: Aeronautics," NASA: http://www.nasa.gov/offices/oct/home/roadmaps/index.html, 2015. 1993.

${ }^{10}$ Ralph, J. A., "Advanced Control for Airbreathing Engines, Volume 1: Pratt and Whitney," NASA CR-189203, July

${ }^{11}$ Connolly, J., Csank, J., Chicatelli, A., and Kilver, J., "Model-Based Control of a Nonlinear Aircraft Engine Simulation using an Optimal Tuner Kalman Filter Approach," AIAA 2013-4002, 2013. 
${ }^{12}$ Scheidler, S. G. and Fottner, L., "Experimental Operating Range Extension of a Twin-Spool Turbofan Engine by Active Stability Control Measures," Transactions of the ASME, Vol. 128, 2006.

${ }^{13}$ Quinn, R., Sims, J., Semega, K. J., and Behbahani, A., "Improved Turbine Engine Performance, Responsiveness, and Prognostics Using Model-Based Control in a Hardware-in-the-Loop Simulation," AIAA 2007-5712, 2007.

${ }^{14}$ Lietzau, K. and Kreiner, A., "Model Based Control Concepts for Jet Engines," ASME GT 2001-0016, 2001.

${ }^{15}$ Behbahani, A., "Perspectives for Distributed Intelligent Engine Controls of the Future," AIAA 2010-6631, 2010.

${ }^{16}$ May, R., Csank, J., Litt, J. S., and Guo, T., "Commercial Modular Aero-Propulsion System Simulation 40K," NASA/TM 2010-216810, 2009.

${ }^{17}$ Luppold, R. H., Gallops, G., Kerr, L., and Roman, J. R., "Estimating In-Flight Engine Performance Variations Using Kalman Filter Concepts," AIAA 1989-2584, 1989.

${ }^{18}$ Espana, M. D., "On the Estimation Algorithm for Adaptive Performance Optimization of Turbofan Engines," AIAA 1993-1823, 1993.

${ }^{19}$ Chatterjee, S. and Litt, J. S., "Online Model Parameter Estimation of Jet Engine Degradation for Autonomous Propulsion Control," AIAA 2003-5425, 2003.

${ }^{20}$ Litt, J. S., "An Optimal Orthogonal Decomposition Method for Kalman Filter-Based Turbofan Engine Thrust Estimation," NASA/TM 2005-213864, 2005.

${ }^{21}$ Viassolo, D. E., Adibhatla, S., Brunell, B. J., Down, J. H., Gibson, N. S., Kumar, A., Mathews, H. K., and Holocomb, L. D., "Advanced Estimation for Aircraft Engines," ACC ThAT05.1, 2007.

${ }^{22}$ Simon, D. L., "A Comparison of Filtering Approaches for Aircraft Engine Health Estimation," Aerospace Science and Technology, Vol. 12, No. 4, 2008, pp. 276-284.

${ }^{23}$ Simon, D. L. and Garg, S., "Optimal Tuner Selection for Kalman Filter-Based Aircraft Engine Performance Estimation," Journal of Engineering for Gas Turbines and Power, Vol. 132, No. 3, 2009.

${ }^{24}$ Simon, D. L. and Rinehart, A. W., "A Model-Based Anomaly Detection Approach for Analyzing Streaming Aircraft Engine Measurement Data," ASME GT 2014-27172, 2014

${ }^{25}$ Simon, D. L., Armstrong, J. B., and Garg, S., "Application of an Optimal Tuner Selection Approach for On-Board Self-Tuning Engine Models," Journal of Engineering for Gas Turbines and Power, Vol. 134, No. 4, 2012.

${ }^{26}$ Csank, J. and Connolly, J. W., "Model-Based Engine Control Architecture with an Extended Kalman Filter," AIAA 2016-1623, 2016.

${ }^{27}$ Martin, S., Wllace, I., and Bates, D. G., "Development and Validation of a Civil Aircraft Engine Simulation Model for Advanced Controller Design," Journal of Engineering for Gas Turbines and Power, Vol. 130, No. 5, June 2008.

${ }^{28}$ May, R. D. and Garg, S., "Reducing Conservatism in Aircraft Engine Response Using Conditionally Active Min-Max Limit Regulators," NASA TM-2012-217814.

${ }^{29}$ Piltan, F., Zare, S., Zadeh, F. S., Mansoorzadeh, M., and Kamgari, M., "Supervised Optimization of Fuel Ratio in IC Engine Based on Design Baseline Computed Fuel Methodology," International Journal Information Technology and Computer Science, Vol. 4, 2013, pp. 76-84.

${ }^{30}$ Panov, V., "Model-Based Control and Diagnostic Techniques for Operational Improvements of Gas Turbine Engines," ETC 10-B098, 2013.

${ }^{31}$ Malapaty, R. and Suresh, M. V. J. J., "Enhanced Fuel Flexibility and Emissions Compliance for Gas Turbines Through Model Based Controls Technology," ASME GTINDIA 2013-3587, 2013.

${ }^{32}$ Csank, J. and Zinnecker, A. M., "Application of the Tool for Turbine Engine Closed-loop Transient Analysis (TTECTrA) for Dynamic Systems Analysis," AIAA 2014-3975, 2014.

${ }^{33}$ Walsh, P. P. and Fletcher, P., Gas Turbine Performance, ASME Press, Blackwell Publishing, NJ, 2nd ed., 2004.

${ }^{34}$ Cline, S. J., Fesler, W., Liu, H. S., Lovell, R. C., and Shaffer, S. J., "High Pressure Compressor Component Performance Report," NASA CR-168245, September 1983.

${ }^{35}$ FAA, "Federal Aviation Regulatoin (FAR): Title 14 Part 33.73 Power or Thrust Response," 2013. 